\title{
L'accessibilité aux aires de jeux dans les parcs de la Communauté métropolitaine de Montréal
}

\author{
Bernardo DE ALVARENGA \\ Centre Urbanisation Culture Société \\ Institut national de la recherche scientifique \\ dealvarengab@ucs.inrs.ca
}

\section{Résumé}

Notre objectif, dans cet article, est de vérifier l'existence d'iniquités environnementales en termes d'accessibilité aux aires de jeux sur le territoire de la Communauté métropolitaine de Montréal pour les enfants, les familles monoparentales, les personnes à faible revenu et les minorités visibles. Deux indicateurs d'accessibilité spatiale sont calculés: une mesure de proximité et une mesure de disponibilité basée sur l'offre et la demande d'aires de jeux. La cartographie des deux indicateurs démontre que, dans les banlieues, ces populations résident plus loin de l'aire de jeux la plus proche (souvent à plus $750 \mathrm{~m}$ ) qui est par ailleurs faiblement saturée (peu d'utilisateurs potentiels dans un rayon de $1000 \mathrm{~m}$ ) alors que, dans le centre de l'agglomération de Montréal, les enfants résident à proximité d'une aire de jeux (souvent à moins de $500 \mathrm{~m}$ ), mais qui est potentiellement saturée. Les résultats des régressions démontrent l'absence d'iniquités flagrantes pour les quatre groupes de population. Toutefois, la population à faible revenu a une accessibilité plus limitée alors que les familles monoparentales bénéficient d'une meilleure accessibilité.

\section{Mots-clés}

Équité environnementale, parcs, aires de jeux, enfants, analyse spatiale, SIG, population à faible revenu, minorités visibles, familles monoparentales, Montréal.

\section{Philippe APPARICIO \\ Centre Urbanisation Culture Société Institut national de la recherche scientifique philippe.apparicio@ucs.inrs.ca}

The accessibility of playgrounds in the Montreal Metropolitan Community's parks

\section{Abstract}

The goal of this paper is to examine the existence of environmental inequities related to the access to playgrounds in the Montreal Metropolitan Community for children, single-parent families, lowincome persons and visible minorities. Two spatial accessibility indicators are computed: one measuring proximity (to the closest playground), another measuring availability (based on supply and demand). The mapping of these two indicators show that suburban populations generally live farther from playgrounds (more than 750 metres) that are not saturated (few potential users within one kilometre). Inversely, in the central parts of the island of Montreal, children usually live closer to playgrounds (less than 500 metres) that are more likely to be saturated (many potential users within one kilometre). The regression results show no major inequities for the four population groups. However, the low-income population has a more limited accessibility to playgrounds, while singleparent families have better accessibility.

\section{Keywords}

Environmental equity, parks, playgrounds, children, spatial analysis, GIS, low-income population, visible minorities, singleparent families, Montreal.

\author{
Anne-Marie SÉGUIN \\ Centre Urbanisation Culture Société \\ Institut national de la recherche scientifique \\ anne-marie.seguin@ucs.inrs.ca
}

La accesibilidad a las áreas de juego en los parques de la Comunidad Metropolitana de Montreal

\section{Resumen}

Nuestro objetivo, en este artículo, es el de verificar la existencia de iniquidades medioambientales sobre la accesibilidad a áreas de juego en el territorio de la Comunidad Metropolitana de Montreal, de niños, familias monoparentales, personas con pocos ingresos y minorías visibles. Dos indicadores de accesibilidad espacial son aquí calculados: una medida de proximidad y otra de disponibilidad según la oferta y la demanda de áreas de juego. La cartografía de los dos indicadores muestra que, en la periferia, esas poblaciones residen lejos del área de juego más cercano (frecuentemente a más de $750 \mathrm{~m}$ ), pero reducidamente saturado (pocos usuarios potenciales por $1000 \mathrm{~m}$ ); aun que, en la aglomeración central de Montreal, los niños residen cerca de un área de juego (frecuentemente a menos de $500 \mathrm{~m}$ ), pero potencialmente saturado. Los resultados de los cálculos de regresión demuestran la ausencia de iniquidades flagrantes para los cuatro grupos de población. Sin embargo, la población con pocos ingresos tiene una accesibilidad más limitada, en tanto que las familias monoparentales benefician de una mejor accesibilidad.

\section{Palabras claves}

Equidad medio-ambiental, parques, áreas de juego, niños, análisis espacial, SIG, población de pocos ingresos, minorías visibles, familias monoparentales, Montreal. 


\section{Introduction}

Les parcs contribuent à la qualité environnementale et au bien-être dans la ville (Chiesura, 2004). La fréquentation d'un parc est susceptible de réduire le stress et l'anxiété (Van Den Berg et al., 2010) et les personnes qui habitent plus près d'un parc tendent à le fréquenter plus souvent et à être plus actives physiquement (Giles-Corti et al., 2005; Kaczynski et al., 2009; Tucker et al., 2009), notamment les jeunes enfants (Veitch et al., 2007). Les parcs constituent des lieux privilégiés pour la rencontre d'autres personnes et la socialisation (Peters et al., 2010); ils peuvent servir aussi de carrefour culturel (Low et al., 2009). Ils ont en outre un rôle important dans l'écosystème urbain, notamment en captant le dioxyde de carbone, en réduisant la température ambiante et en absorbant les eaux de ruissellement (Heckert et Rosan, 2016).

En raison des bénéfices qu'ils apportent, nombreuses sont les municipalités québécoises qui ciblent les parcs urbains dans leur plan d'urbanisme, comme éléments importants pour améliorer la qualité de vie des citoyens au sein des quartiers. D’ailleurs, le gouvernement du Québec, par la Loi sur l'aménagement et l'urbanisme, autorise les municipalités à demander une contribution aux fins de parcs correspondant jusqu'à $10 \%$ de la superficie d'un terrain (en espèces ou en nature) pour tout projet de développement ou de redéveloppement immobilier (Fontaine, 2011). La Ville de Montréal, dans sa politique familiale, par exemple, s'engage à offrir « un espace de verdure ou un parc à courte distance de marche, propre et bien entretenu » (2008: 5). En 2010, elle s'est dotée du Programme d'amélioration des aménagements des parcs (PAAP), un plan triennal pour investir dans les installations existantes de parcs.

Reconnaissant leurs bienfaits, plusieurs auteurs se sont intéressés à la répartition des parcs au sein des métropoles du point de vue de l'équité environnementale, mais les résultats sont variables selon les contextes urbains et selon les populations étudiées. Certaines études démontrent que des groupes - personnes à faible revenu et minorités ethnoculturelles - ont une accessibilité plus faible aux parcs et à leurs équipements, notamment dans plusieurs villes étasuniennes (Talen et Anselin, 1998; Loukaitou-Sideris et Stieglitz, 2002; Cradock et al., 2005; Sister et al., 2010). D'autres concluent à l'absence de corrélation entre la distribution spatiale de ces groupes et celle des parcs, voire à une situation plus favorable (Ellaway et al., 2007; Abercrombie et al., 2008; Jones et al., 2009; Apparicio et al., 2010).
Dans cet article sur Montréal, nous nous intéresserons plus particulièrement aux aires de jeux, une autre étude (Apparicio et al., 2010) s'étant déjà penchée sur la distribution des parcs montréalais. Notre objectif est de poser un diagnostic d'équité environnementale relativement à l'accessibilité aux aires de jeux sur le territoire de la Communauté métropolitaine de Montréal (CMM) en fonction de différentes populations, notamment celle des enfants, ce qui n'a pas été fait à ce jour, mais aussi des personnes à faible revenu, des minorités visibles et des familles monoparentales.

\section{Importance des parcs et des aires de jeux pour les enfants}

De nombreuses études ont démontré les bénéfices des parcs en termes d'activité physique pour les enfants. Tappe et al. (2013) concluent que la proximité des parcs et la «marchabilité » pour s'y rendre sont importantes pour favoriser l'activité physique des enfants âgés de 6 à 11 ans. Roemmich et al. (2006) et Timperio et al. (2008) ont aussi signalé l'existence d'une corrélation entre la proximité des aires de jeux et l'activité physique des enfants. Malgré ces bienfaits, certains auteurs ont démontré que les enfants ne bénéficient pas toujours d'une bonne accessibilité aux parcs. Par exemple, à Phoenix, Cutts et al. (2009) affirment que les jeunes de moins de 18 ans sont sous-représentés dans les quartiers offrant un meilleur potentiel piétonnier et une bonne accessibilité aux parcs.

Les parcs permettant d'augmenter les niveaux d'activité physique des enfants, ils pourraient servir dans la lutte à l'obésité chez les jeunes. D'ailleurs, une étude longitudinale menée à Los Angeles met en évidence le fait que les enfants de 9 ou 10 ans qui avaient habité à $500 \mathrm{~m}$ ou moins d'un parc présentaient, à l'âge de 18 ans, un indice de masse corporelle (IMC) plus bas que ceux qui avaient résidé à plus de $500 \mathrm{~m}$ (Wolch et al., 2011). Dans la même veine, l'étude de Veugelers et al. (2008) et celle Singh et al. (2010) ont montré une relation négative entre la proximité des parcs et l'obésité chez les enfants. Quant à Potwarka et al. (2008), ils arrivent aussi à ce constat en ne retenant que les aires de jeux. En revanche, Poole et Moon (2017) ne voient aucune relation entre l'obésité des enfants âgés de 4 à 5 ans et la proximité des aires de jeux de leur résidence, résultats corroborés par d'autres études (Burdette et Whitaker, 2004; Lovasi et al., 2011). 
Les bénéfices des parcs pour les enfants ne se limitent pas à l'activité physique. En effet, plusieurs auteurs ont démontré que les parcs, et plus particulièrement les aires de jeux, en incitant au jeu, peuvent avoir un rôle important dans le développement moteur, cognitif et social des enfants (exploration de nouveaux lieux, partage d'équipements, etc.) (Czalczynska-Podolska, 2014; Miller et al., 2017).

Outre ces effets potentiels sur les niveaux d'activité physique, sur l'obésité et sur le développement des enfants, les parents de jeunes enfants semblent accorder une importance particulière à l'accessibilité spatiale aux aires de jeux, surtout dans les quartiers centraux. À Montréal, l'étude de Wiebe (2010) confirme que les aires de jeux constituent le service de proximité le plus important pour les parents de l'arrondissement Rosemont-La PetitePatrie participant à son étude. Sallis et al. (1997) soulignent que, dans le choix d'une aire de jeux par les parents, sa proximité immédiate est un critère très important.

\section{Études antérieures sur l'équité environne- mentale et les aires de jeux}

Les travaux s'inscrivant dans le courant de recherche de l'équité environnementale (aussi nommée justice distributionnelle) s'intéressent aux situations de surexposition à des nuisances (bruit, pollution de l'air, etc.) ou encore de plus faible accessibilité aux éléments positifs du cadre de vie urbain (végétation, parcs, commerces alimentaires, etc.) que vivent certains groupes de population (par exemple définis par leur niveau de revenu, leur âge, leur appartenance ethnique) (Walker, 2012; Séguin et Apparicio, 2013).

S'il existe de nombreuses études dans le domaine de l'équité environnementale sur les parcs (dont Wolch et al., 2005; Boone et al., 2009; Maroko et al., 2009; Apparicio et al., 2010; Sister et al., 2010), peu d'auteurs se sont attardés spécifiquement aux aires de jeux. Seules quatre études l'ont fait dans une optique d'équité (Talen et Anselin, 1998; Smoyer-Tomic et al., 2004; Cradock et al., 2005; Ellaway et al., 2007), dont une sur une ville canadienne (Smoyer-Tomic et al., 2004).

L'étude de Talen et Anselin (1998) est extrêmement intéressante sur le plan des indicateurs proposés. Les auteurs y comparent plusieurs mesures pour évaluer l'accessibilité aux aires de jeux. Ils remettent en question l'approche «conteneur» souvent utilisée dans le passé, où l'accessibilité est mesurée uniquement en comptabilisant les services compris dans une entité spatiale (un secteur de recensement le plus souvent). Ils la comparent avec d'autres mesures d'accessibilité plus précises, comme la distance minimale de l'aire de jeux, la distance moyenne de l'ensemble des aires de jeux et un modèle gravitaire. Sur le plan méthodologique, ils concluent que la distance pour se rendre à l'aire de jeux la plus proche est la mesure la plus intéressante. Sur le plan empirique, leur étude montre que les secteurs caractérisés par une forte accessibilité aux aires de jeux sont aussi ceux ayant une faible proportion de jeunes, reflétant ainsi un mauvais appariement entre l'offre d'aires de jeux et la demande potentielle.

Smoyer-Tomic et al. (2004) suivent la recommandation de Talen et Anselin (1998) en employant, dans leur étude, la mesure de la distance minimale des aires de jeux à Edmonton. Ils concluent que les aires de jeux pour enfants dans les parcs sont distribuées équitablement, c'est-à-dire que les zones ayant les besoins sociaux les plus grands (mesurés à partir de cinq variables) ${ }^{1}$ sont celles qui ont la plus grande accessibilité. Par contre, quand les auteurs retiennent uniquement les aires de jeux considérées comme étant de bonne qualité par la Ville d'Edmonton, cette relation ne tient plus.

Cradock et al. (2005) et Ellaway et al. (2007) arrivent à des conclusions similaires quant à l'accessibilité et à la qualité des aires de jeux. À Boston, Cradock et al. (2005) signalent que les îlots où résident le plus de jeunes enfants sous le seuil de pauvreté sont situés plus près des aires de jeux, mais que ces dernières sont moins sécuritaires. À Glasgow, Ellaway et al. (2007) concluent que les zones de code postal avec les plus hauts indices de pauvreté ont plus d'aires de jeux, mais qu'elles sont de plus piètre qualité.

Coen et Ross (2006) font une analyse exclusivement de la qualité des aires de jeux dans six territoires de centres locaux de services communautaires (CLSC) de l'île de Montréal sélectionnés sur la base d'indicateurs de santé (entre autres l'espérance de vie): deux avec des valeurs faibles (Hochelaga-Maisonneuve et Saint-Henri), deux moyennes (Rosemont et Ahuntsic) et deux élevées (NotreDame-de-Grace-Montréal-Ouest et Westmount). Quant à la qualité des aires de jeux, elle est mesurée à partir de données d'observation. Elles révèlent, photographies

1 Pourcentages d'enfants de moins de 15 ans, de personnes à faible revenu, de ménages non motorisés, de ménages dans des appartements ou des maisons en rangée et des personnes résidant depuis moins de 5 ans à la même adresse. 
à l'appui, des variations importantes dans cette qualité, qui semble plus médiocre dans les territoires de CLSC avec de faibles valeurs sur les indicateurs de santé. D'autres chercheurs arrivent toutefois à des résultats contraires dans un autre contexte géographique: Poole et Moon (2017) affirment qu'à Southampton, les enfants dans les zones avec des niveaux de pauvreté élevés ont accès à des aires de jeux de meilleure qualité, en raison du ciblage de ces secteurs par les autorités municipales.

Finalement, pour évaluer l'accessibilité à un service donné, plusieurs auteurs préconisent de tenir compte non seulement de l'offre, mais aussi de la demande. Pour ce faire, les mesures les plus connues sont les modèles gravitaires et les méthodes Two-Step Floating Catchment Area (2SFCA), largement utilisés pour mesurer l'accessibilité aux services de santé (Ngui et Apparicio, 2011; Bell et al., 2013). Plusieurs raisons peuvent justifier le recours à ces modèles dans le cadre d'une étude sur l'accessibilité aux aires de jeux. Plus il y a d'habitants autour d'une aire de jeux, plus celle-ci est susceptible de recevoir un volume d'utilisateurs important pouvant conduire à une dégradation accélérée des installations, ou encore d'être saturée, c'est-à-dire d'être occupée par un nombre élevé d'utilisateurs simultanés à certains moments, réduisant ainsi leur attrait. Par exemple, des études à Baltimore et Los Angeles signalent que, comparativement à la population blanche, les minorités visibles résident plus près des parcs, qui sont toutefois plus saturés, c'est-à-dire avec un grand nombre d'utilisateurs potentiels dans leur environnement immédiat (Boone et al., 2009; Sister et al., 2010).

À la lumière de ces études portant sur l'accessibilité aux aires de jeux pour enfants dans un contexte d'équité environnementale, notre objectif principal dans cet article est de vérifier si les aires de jeux pour enfants sont équitablement distribuées sur le territoire de la Communauté métropolitaine de Montréal (CMM). Nous nous pencherons sur quatre groupes de population-les enfants, les familles monoparentales, les personnes appartenant aux minorités visibles et les personnes à faible revenu - pour vérifier s'ils ont ou non un accès plus limité. Nous avons retenu ces groupes pour différentes raisons. Premièrement, les aires de jeux étant habituellement destinées aux enfants de 2 à 12 ans, mesurer l'accessibilité pour les enfants s'impose en toute logique. Toutefois, quel que soit le découpage géographique retenu, les données sur les groupes d'âge dans le recensement de 2011 étant regroupées par tranche de 5 ans ( $0-4,5-9,10-14$ ans), nous avons sélectionné uniquement les deux premières tranches d'âge, soit les enfants de moins de 10 ans. Deuxièmement, les personnes à faible revenu et les minorités visibles sont plus susceptibles de vivre des situations d'iniquité environnementale, selon la littérature dans ce domaine (Carrier et al., 2016), notamment quant à l'accessibilité aux parcs (Talen et Anselin, 1998; Loukaitou-Sideris et Stieglitz, 2002; Cradock et al., 2005; Abercrombie et al., 2008). Or, dans une logique d'équité compensatoire, selon Sister et al. (2010), ces groupes devraient bénéficier d'un meilleur accès, notamment en raison du manque d'espaces extérieurs, publics ou privés, pour le jeu et d'options abordables pour des activités récréatives dans les quartiers où se concentrent ces deux populations. Quant aux familles monoparentales, elles sont rarement étudiées dans les travaux sur l'équité environnementale. Pourtant, en raison de la lourdeur de la responsabilité des enfants qui repose sur un seul adulte, il paraît important de vérifier si ces familles sont localisées près des parcs, afin d'en faciliter l'utilisation par les enfants qui les composent. II s'agit là d'une des contributions de cette étude.

\section{Méthodologie}

\section{Territoire d'étude et données}

Le territoire d'étude est la CMM, qui regroupe 82 municipalités (figure 1). Ce territoire se décompose en cinq grandes sous-régions: l'agglomération de Montréal, qui comprend la ville-centre de la région métropolitaine (Montréal), la Ville de Laval, la couronne nord, l'agglomération de Longueuil et le reste de la couronne sud.

Nous avons retenu les parcs comprenant au moins une aire de jeux, soit un total de 2169 parcs comprenant 4256 aires de jeux pour enfants. En raison de l'absence de données relatives à la qualité des aires de jeux et vu leur nombre élevé sur le territoire d'étude, la dimension de la qualité des aires de jeux n'est pas abordée dans le cadre de cette étude. La localisation des parcs et des aires de jeux a été réalisée et validée à partir de plusieurs sources: les données géomatiques fournies par les MRC ou municipalités; les sites Web des municipalités; les services Google Maps et ses images satellitaires; mais aussi, des visites sur terrain afin de clarifier quelques incohérences entre les différentes sources utilisées. Par la suite, dans un logiciel de système d'information géographique (SIG) (ArcGIS), nous avons ajouté un point au centre de chaque module d'aire de jeux distinct dans un parc. 


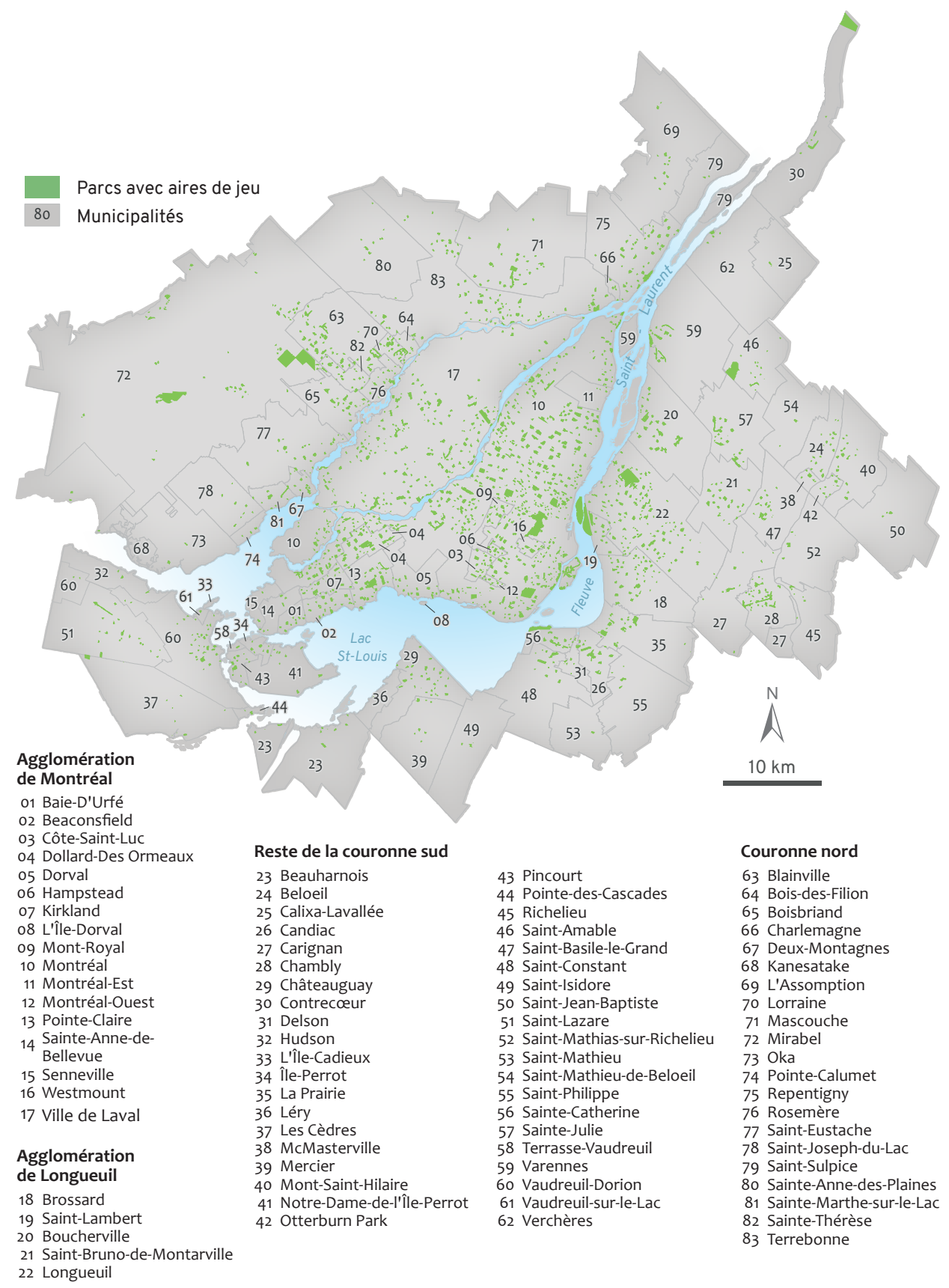

FIGURE 1 Territoire d'étude: la Communauté métropolitaine de Montréal | Conception: Alvarenga, Apparicio et Séguin, 2018 Adaptée par le Département de géographie de l'Université Laval, 2019 
Puis, nous avons eu recours à la carte d'utilisation du sol produite par la CMM pour l'année 2012 afin d'extraire uniquement les parties occupées à des fins résidentielles de chacun des 26584 îlots de diffusion du recensement de 2011. II est à noter que, pour ces îlots, Statistique Canada diffuse uniquement trois variables, soit la population totale, le nombre de logements occupés et le nombre de ménages. Concernant les données sociodémographiques, cinq variables ont été extraites à l'échelle des secteurs de recensement (environ 5000 habitants en moyenne) et non des aires de diffusion (environ 600 habitants) le nombre d'enfants de moins de 10 ans, le nombre de familles monoparentales, le nombre de personnes à faible revenu et le nombre de personnes appartenant à une minorité visible. Nous n'avons pas utilisé les données sociodémographiques à l'échelle des aires de diffusion, soit une démarche habituelle lorsqu'on souhaite un découpage spatial le plus précis possible. Étant donné le caractère non obligatoire de l'enquête nationale auprès des ménages de 2011, les données ne sont guère fiables à cette échelle. Par conséquent, nous avons retenu le découpage des secteurs de recensement. II est à noter que, pour trois municipalités de la CMM, aucune donnée n'est disponible sur la base des secteurs de recensement; ces municipalités ont donc été écartées de notre analyse (Calixa-Lavallée, Contrecœur et Saint-Jean-Baptiste). ${ }^{2}$ Pour d'autres municipalités, comme Saint-Isidore, Saint-Mathieu et une grande partie de Saint-Constant, les données étaient soit absentes soit non fiables, selon Statistique Canada. Ces municipalités ont donc été également exclues de l'étude (voir les figures 2 et 3 où sont représentées les zones non étudiées).

\section{Mesurer l'accessibilité aux aires de jeu}

Tel que mentionné plus haut, à l'aide de la carte d'utilisation du sol de la CMM, nous avons retenu uniquement les parties des îlots de diffusion ayant une fonction résidentielle en 2011, afin d'établir le centroïde résidentiel de chaque îlot. Dans cette étude, nous examinons l'accessibilité des aires de jeux destinées aux enfants âgés de moins de 10 ans. Par la suite, à l'instar de Pham et al. (2012), nous avons estimé le nombre d'enfants de moins de 10 ans dans chaque îlot comme suit:

$$
p_{i}=t_{i} \frac{E_{j}}{T_{j}}
$$

2 Cela s'explique par le fait que ces municipalités ne sont pas comprises dans la région métropolitaine de recensement de Montréal telle que définie par Statistique Canada. où $p_{i}$ est le nombre estimé d'enfants de moins de 10 ans dans l'îlot de diffusion $i$ compris dans le secteur de recensement $j, t_{i}$ est la population totale de l'îlot de diffusion, $E_{j}$ et $T_{j}$ sont respectivement le nombre d'enfants et la population totale dans le secteur de recensement $j$.

Deux mesures d'accessibilité sont mises en œuvre dans le SIG à partir de l'extension Network Analyst d'ArcGIS à partir de la distance réticulaire (à travers le réseau de rues): la distance à l'aire de jeux la plus proche et une mesure de disponibilité basée sur l'offre et la demande de parcs, la méthode du Enhanced Two-Step Floating Catchment Area (E2SFCA).

Pour évaluer la proximité immédiate de l'aire de jeux j, nous avons, dans un premier temps, rattaché les centroïdes des aires de jeux au segment de rue le plus proche, dénommé j'. Puis, nous avons calculé, pour chaque centroïde $i$ des îlots résidentiels, le chemin le plus court à travers le réseau de rues excluant les autoroutes $\left(d_{i j}\right)$. À cette distance, nous avons ajouté la distance euclidienne entre le point $j$ ' et l'aire de jeux $j$, correspondant globalement à la distance de marche à travers le parc entre la rue et l'aire de jeux $\left(d_{j j}\right)$. Autrement dit, pour chaque îlot, la distance de marche jusqu'au parc le plus proche $\left(M_{i}\right)$ exprimée en mètres est calculée de la manière suivante:

$$
M_{i}=\min \left|d_{i j^{\prime}}+d_{j^{\prime}}\right|
$$

À l'instar de plusieurs auteurs (Hodgson et Hewko, 2003; Apparicio et al., 2017), cette mesure d'accessibilité obtenue au niveau des îlots de diffusion $\left(M_{i}\right)$ a ensuite été agrégée au niveau de chaque secteur de recensement $\left(M_{\mathrm{s}}\right)$ pour limiter les erreurs d'agrégation. Pour ce faire, nous calculons simplement la moyenne pondérée par la population de moins de 10 ans $\left(w_{i}\right)$ pour les îlots inclus dans le secteur de recensement $s$ :

$$
M_{S}=\frac{\sum_{i \in S} w_{i} M_{i}}{\sum_{i \in S} w_{i}}=\frac{\sum_{i \in S} w_{i}\left(\min \left|d_{i j^{\prime}}+d_{j^{\prime}}{ }^{\prime}\right|\right)}{\sum_{i \in S} w_{i}}
$$

La méthode du 2SFCA a été proposée par Luo et Wang (2003). Tel que son nom l'indique, elle se décompose en deux étapes. Dans la première, il s'agit de calculer un ratio pour chaque aire de jeux:

$$
R_{j}=\frac{S_{j}}{\sum_{i \in\left\{d_{i j} \leq d_{0}\right\}} p_{i} / 1000}
$$


où $R_{j}$ le ratio offre-demande pour l'aire de jeux $j$ dans l'aire de desserte définie par $d_{0}, d_{i j}$ est la distance réticulaire entre l'îlot de diffusion et l'aire de jeux $j, d_{0}$ est la distance réticulaire pour définir l'aire de desserte $(1000 \mathrm{~m}$, par exemple), $p_{i}$ est la population d'enfants de moins de 10 ans dans l'îlot $i, S_{j}$ est une pondération accordée en $j$. Ce dernier paramètre est très important pour des services de santé; par exemple, il pourrait être le nombre de lits pour des hôpitaux ou encore le nombre de médecins par clinique médicale. Dans le cas des parcs, il pourrait être le nombre d'hectares ou d'équipements. Dans le cas qui nous concerne ici, les aires de jeux, la pondération a été mise à 1 puisque nous ne disposons pas de ces informations. De plus, on peut supposer que leur superficie varie peu d'une aire de jeux à l'autre. Au final, ce ratio initial $R_{j}$ exprime l'inverse du nombre d'enfants de moins de 10 ans résidant à moins de $1000 \mathrm{~m}\left(d_{0}\right)$ de l'aire de jeux $j$ à travers le réseau de rues. Dans une deuxième étape, pour chaque centroïde de l'îlot $i$, nous sélectionnons les aires de jeux situées à moins de $n$ de distance réticulaire $\left(d_{0}\right)$ et faisons la somme des ratios initiaux $R_{i}$ :

$$
A_{i}=\sum_{i \in\left\{d_{i j} \leq d_{0}\right\}} R_{j}=\sum_{i \in\left\{d_{i j} \leq d_{0}\right\}} \frac{S_{j}}{\sum_{i \in\left\{d_{i j} \leq d_{0}\right\}} p_{i} / 1000}
$$

Si la valeur de mesure d'accessibilité pour l'îlot $i\left(A_{i}\right)$ est égale à 0 , alors il n'y a pas d'aire de jeux à une distance $d_{0}$ de l'îlot. Plus la valeur de $A_{i}$ est élevée, meilleure est l'accessibilité: plus il y a d'aires de jeux pour 1000 enfants dans un rayon de $1000 \mathrm{~m}\left(d_{0}\right)$, soit une disponibilité élevée en fonction de l'offre et de la demande. À l'inverse, plus cette valeur est faible, plus le ratio offre-demande est faible; autrement dit, plus les aires de jeux à une distance $d_{0}$ sont potentiellement saturées.

L'évaluation de l'accessibilité est dichotomique puisqu'elle présuppose qu'il n'y a pas d'accessibilité au-delà du seuil de distance retenu $\left(d_{i j}>d_{0}\right)$ et que tous les habitants résidant dans l'aire de desserte $\left(d_{i j}<d_{0}\right)$ bénéficient de la même accessibilité, quelle que soit la distance les séparant du service. Par conséquent, Luo et Qi (2009) ont proposé une version améliorée (E2SFCA) en divisant la zone de desserte en trois zones: par exemple, moins de 10 minutes $\left(d_{1}\right), 10$ à 20 minutes $\left(d_{2}\right)$, et 20 à 30 minutes $\left(d_{3}\right)$. Pour chacune de ces trois zones, il est alors possible d'appliquer une pondération $\left(W_{k}\right)$ avec une fonction gaussienne. La méthode E2SFCA s'écrit alors:

$$
\begin{aligned}
& R_{j}=\frac{S_{j}}{\sum_{i \in\left\{d_{i j} \leq d_{0}\right\}} p_{i}}= \\
& \frac{S_{j}}{\sum_{i \in\left\{d_{i j} \leq d_{1}\right\}} p_{i} W_{1}}+\frac{S_{j}}{\sum_{i \in\left\{d_{i j} \leq d_{2}\right\}} p_{i} W_{2}}+\frac{S_{j}}{\sum_{i \in\left\{d_{i j} \leq d_{3}\right\}} p_{i} W_{3}} \\
& A_{i}=\sum_{i \in\left\{d_{i j} \leq d_{0}\right\}} R_{j}= \\
& \sum_{i \in\left\{d_{i j} \leq d_{1}\right\}} R_{j} W_{1}+\sum_{i \in\left\{d_{i j} \leq d_{2}\right\}} R_{j} W_{3}+\sum_{i \in\left\{d_{i j} \leq d_{3}\right\}} R_{j} W_{3}
\end{aligned}
$$

où les valeurs de $W_{1}, W_{2}, W_{3}$ sont respectivement égales à 1 , 0,68 et 0,22 avec une fonction gaussienne de décroissance lente et 1,0,42 et 0,09 avec une fonction gaussienne de décroissance rapide. De plus, d'autres auteurs (McGrail et Humphreys, 2009; Wan et al., 2012) ajoutent une quatrième zone (30 à 60 minutes). Les pondérations sont alors égales à $1,0,8,0,55$ et 0,15 avec une fonction gaussienne de décroissance lente, et à 1, 0,6, 0,25 et 0,05 avec une fonction gaussienne de décroissance rapide. Par conséquent, trois paramètres doivent être établis pour calculer la méthode du E2SCFA: la limite des zones de desserte $\left(d_{0}\right)$, le nombre de zones ( 3 ou 4 ) et les pondérations selon une fonction de décroissance rapide ou lente.

Quant aux limites des zones de service des aires de jeux pour I'E2SCFA, ces valeurs ne sont pas établies dans la littérature. En effet, dans les études portant sur l'accessibilité aux parcs, plusieurs seuils de distance sont retenus. SmoyerTomic et al. (2004) ont sélectionné une distance de $800 \mathrm{~m}$. Karsten (2002) utilise les seuils de distance suivants à Amsterdam: 100, 400 et 800 mètres selon le type de parc. Finalement, d'autres études utilisent un seuil de distance que leurs auteurs estiment confortable pour la marche d'un enfant: de 10 à 15 minutes, soit autour de 1000 m (Potwarka et al., 2008; Poole et Moon, 2017). II n'y a donc pas de consensus quant au choix des seuils de distance.

Par conséquent, dans le cadre de cette étude, nous avons retenu quatre seuils de distance: moins de $250 \mathrm{~m}$, de 250 à 499 , de 500 à 749 et de 750 à 1000 . Au-delà d'un kilomètre, nous estimons qu'il est peu probable que l'enfant se rende à pied à l'aire de jeux. De plus, nous avons choisi des pondérations selon une fonction de décroissance lente. Finalement, une fois la mesure du E2SFCA obtenue pour les îlots de diffusion, elle est ramenée au niveau des secteurs 
de recensement par un simple calcul de la moyenne des valeurs du E2SFCA pour les îlots compris dans le secteur de recensement, tel que préconisé par Bell et al. (2013).

Une fois ces deux mesures obtenues - distance jusqu'à l'aire de jeux la plus proche (accessibilité immédiate) et l'E2SFCA (tenant compte à la fois de l'offre et de la demande) -, il est possible de les croiser afin de déterminer les secteurs les plus avantagés ou les plus désavantagés selon ces deux mesures d'accessibilité. Pour ce faire, nous avons divisé chaque mesure en cinq catégories: pour la distance minimale, de 0 à $249 \mathrm{~m}$, de 250 à 499, de 500 à 749, de 750 à 999 et de $1000 \mathrm{~m}$ et plus; et en quintiles pour la mesure du E2SCFA. Nous obtenons ainsi une matrice comprenant cinq lignes et cinq colonnes, dans laquelle il est possible de déterminer les secteurs de recensement selon les combinaisons de faible, moyenne ou bonne accessibilité à partir des deux indicateurs. Il est à noter que cette démarche a été récemment proposée et appliquée à Montréal par Apparicio et al. (2016).

\section{Évaluer les iniquités environnementales}

Une fois les deux mesures d'accessibilité calculées, elles peuvent être introduites comme variables dépendantes dans des modèles de régression linéaire multiple. Quant aux variables indépendantes, elles comprennent, dans un premier temps, des variables dichotomiques pour quatre zones de la CMM - Laval, couronne nord, Longueuil et le reste de la couronne sud - afin de vérifier si l'accessibilité y est significativement différente comparativement à l'agglomération (île) de Montréal (catégorie de référence). Dans un second temps, nous avons introduit les groupes de population pour lesquels nous voulons vérifier l'existence d'équité environnementale en termes d'accessibilité aux aires de jeux: les familles monoparentales, les familles à faible revenu et la population de minorités visibles, sous forme de pourcentage par secteur de recensement, et les enfants en intégrant la variable de la densité d'enfants de moins de 10 ans par secteur. II est à noter que la densité d'enfants n'est pas introduite dans le second modèle puisque l'E2SFCA tient compte de la présence d'enfants, ce qui génère des problèmes d'endogénéité. Nous privilégions la densité d'enfants dans les zones résidentielles des secteurs de recensement plutôt que le pourcentage d'enfants, car afin d'éviter la congestion des équipements, on peut supposer que les administrations municipales installent plus d'aires de jeux dans les zones avec une grande densité d'enfants. De plus, selon Sister et al. (2010), les groupes défavorisés résident dans les zones plus denses où l'on trouve moins d'autres espaces ouverts ou informels pour le jeu. L'ajout de la variable de densité d'enfants nous permet alors de vérifier l'existence d'iniquités dans l'accessibilité aux parcs pour les familles monoparentales, les personnes à faible revenu et les minorités visibles, une fois cette variable contrôlée.

Finalement, un dernier modèle est réalisé - cette fois-ci une régression logistique multinomiale - avec, comme variable dépendante, les catégories issues du croisement des deux variables d'accessibilité. De la sorte, on verra si plus les pourcentages des trois groupes de population et la densité d'enfants augmentent, plus la probabilité d'appartenir à l'une ou l'autre des catégories d'accessibilité augmente ou diminue. L'ensemble de ces modèles a été réalisé dans SAS version 9.3.

\section{Résultats}

\section{L'accessibilité aux aires de jeux dans la région de Montréal}

Les statistiques descriptives des deux indicateurs d'accessibilité pondérées par la population d'enfants de moins de 10 ans sont reportées au tableau 1 pour les 881 secteurs de recensement du territoire. Les valeurs des moyennes et des médianes montrent que, globalement, les enfants ont une bonne accessibilité aux aires de jeux sur le territoire de la CMM. Concernant la proximité immédiate, les enfants sont en moyenne à un peu plus de $500 \mathrm{~m}$ de l'aire de jeux la plus proche (moyenne $=559$; médiane $=512$ ). Toutefois, les valeurs percentiles extrêmes signalent que respectivement $10 \%$ et $5 \%$ des enfants résident à près de $800 \mathrm{~m}$ et à plus de 1000 de l'aire de jeux la plus proche. Concernant la disponibilité en fonction de l'offre et de la demande (E2SFCA), on trouve en moyenne, sur le territoire de la CMM, 26 aires de jeux pour 1000 enfants, soit une aire pour 38 enfants dans un rayon de $1000 \mathrm{~m}$ de distance réticulaire. 
a. Proximité de l'aire de jeu la plus proche Distance réticulaire (en mètres)

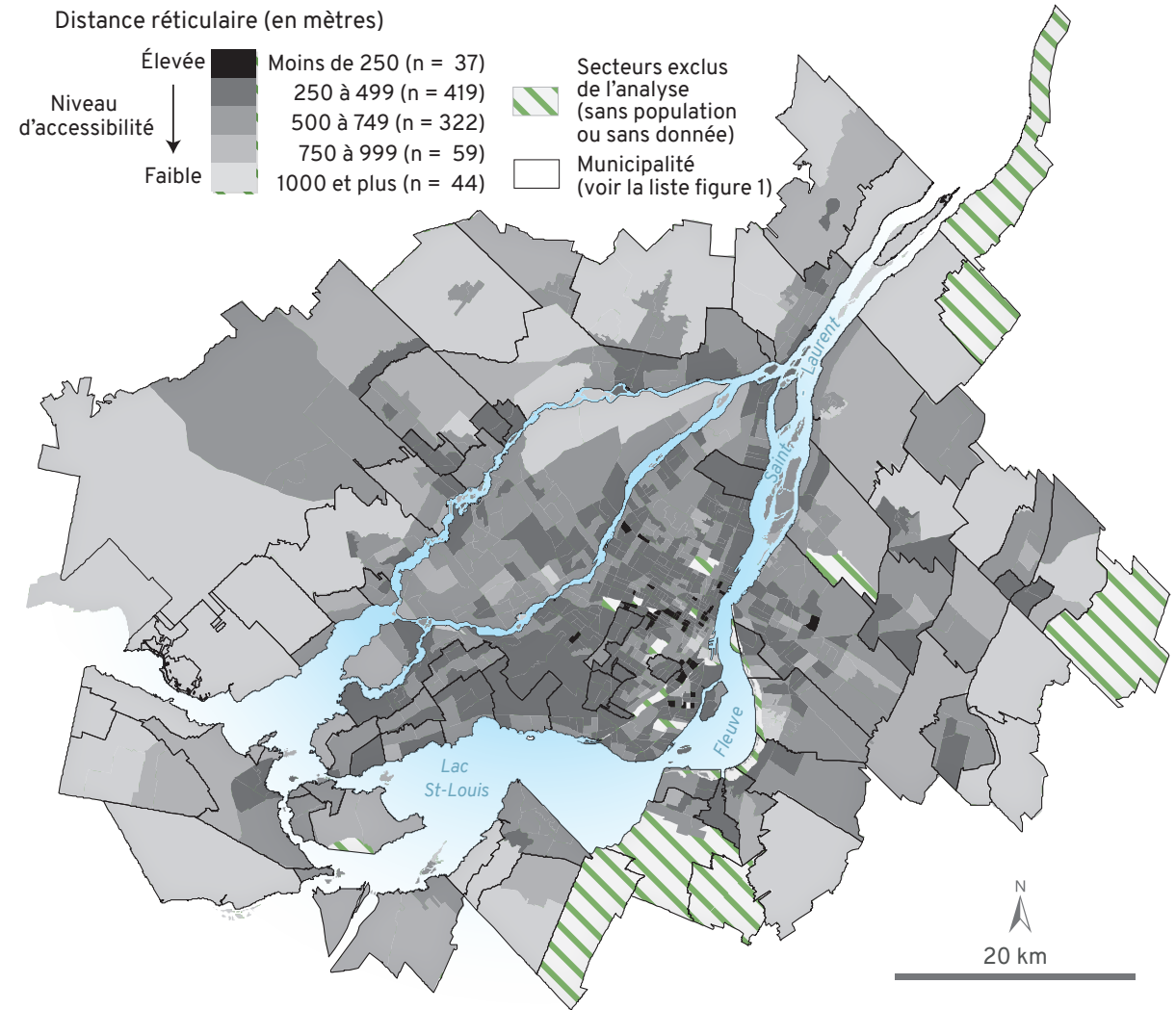

b. Disponibilité potentielle dans un rayon de $1000 \mathrm{~m}$ Disponibilité potentielle (aires de jeu pour 1000 enfants)

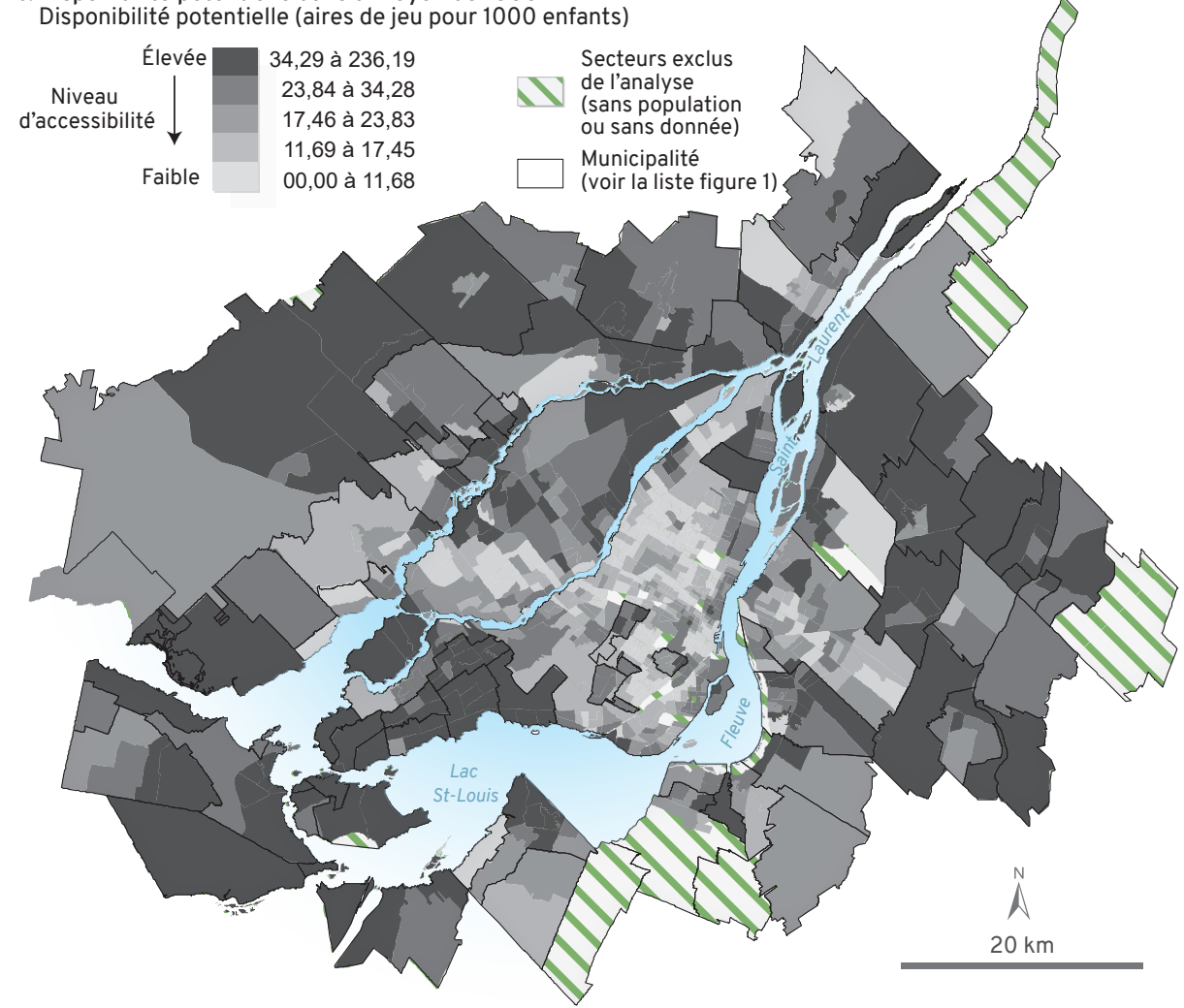

FIGURE 2 Cartographie des deux indicateurs d'accessibilité aux aires de jeux par secteur de recensement | Conception: Alvarenga, Apparicio et Séguin, 2018 Adaptée par le Département de géographie de l'Université Laval, 2019 
TABLEAU 1 Statistiques descriptives des indicateurs d'accessibilité pondérés par la population d'enfants de moins de 10 ans par secteur de recensement Conception: Alvarenga, Apparicio et Séguin, 2018

\begin{tabular}{|l|c|c|c|c|c|c|c|c|c|}
\hline & N & Moyenne & P5 & P10 & Q1 & Q2 & Q3 & P90 & P95 \\
\hline Aire de jeux la plus proche & 881 & 559 & 282 & 327 & 408 & 512 & 640 & 799 \\
\hline E2SFCA & 881 & 25,94 & 6,46 & 9,09 & 13,63 & 21,39 & 33,10 & 48,56 & 58,50 \\
\hline
\end{tabular}

P5, P10, P90, P95: percentiles. Q1, Q2, Q3: quartiles.

À la lecture de la figure 2, il est clair que ces deux indicateurs d'accessibilité varient passablement à travers le territoire de la CMM, mais de manière différente selon l'indicateur. En ce qui concerne la proximité à l'aire de jeu la plus proche, la quasi-totalité des secteurs de recensement de l'agglomération de Montréal présentent des distances de l'aire de jeux la plus proche inférieures à $500 \mathrm{~m}$, alors que les secteurs situés à plus d'un kilomètre d'une aire de jeux sont très majoritairement localisés dans les couronnes nord et sud de la CMM (figure 2a).

Quant à la disponibilité potentielle dans un rayon de $1000 \mathrm{~m}$, la carte des quintiles de disponibilité des aires de jeux selon l'E2SCFA (figure 2b) contraste fortement avec la carte de proximité décrite précédemment, telle une image inversée, mais avec des exceptions un peu partout sur le territoire. Globalement, l'accessibilité semble meilleure dans les couronnes nord et sud, et plus faible dans l'agglomération de Montréal sauf dans l'ouest (notamment dans les municipalités allant de Dorval à Senneville). Il est à noter que l'accessibilité est aussi plus faible dans l'ouest de Laval et à Longueuil et dans quelques petites enclaves dispersées dans les couronnes nord et sud. Cela signifie que les parcs y sont donc potentiellement saturés. En guise d'exemple, au centre de l'île, les secteurs appartenant au premier quintile de l'E2SFCA (en gris pâle) ont des valeurs inférieures à 11,84 aires de jeux pour 1000 enfants (soit plus de 84 enfants par aire) alors que les secteurs appartenant au dernier quintile (gris foncé) ont des valeurs supérieures à 34,47 aires de jeux pour 1000 enfants (soit moins de 29 enfants par aire).

En résumé, dans le centre de lîle, les enfants vivent à proximité d'une aire de jeux, mais celle-ci est susceptible d'avoir beaucoup d'utilisateurs dans un rayon de $1000 \mathrm{~m}$ à travers le réseau de rues alors que, dans les couronnes nord et sud, les enfants résident plus loin d'une aire de jeux, mais avec peu d'utilisateurs potentiels dans de très nombreux secteurs.

Les résultats du croisement de deux indicateurs d'accessibilité (figure $2 a$ et b) sont cartographiés à la figure 3. Cinq types de secteurs de recensement sont ainsi obtenus. Près de $20 \%$ des 881 secteurs $(n=174)$ en gris très foncé sont caractérisés par une bonne accessibilité selon les deux indicateurs: ils sont situés à moins de 500 m d'une aire de jeux avec une faible saturation potentielle (moins de 42 enfants par aire de jeux dans un rayon de $1000 \mathrm{~m}$ ). Ces secteurs ne suivent pas un patron de distribution très net. Ils sont répartis sur tout le territoire. Au sein de l'agglomération de Montréal, on note une présence un peu plus marquée dans les portions ouest et nord-est (le long du fleuve Saint-Laurent) de l'île de Montréal. On en trouve aussi dispersés dans des secteurs de Laval et des municipalités des couronnes nord et sud.

À l'autre extrémité, on trouve uniquement 46 secteurs (5\%) en gris très pâle avec une faible accessibilité selon les deux indicateurs: éloignés d'une aire de jeux (à plus de $750 \mathrm{~m}$ ) et avec un niveau de saturation élevé (plus de 57 enfants par aire de jeux dans un rayon de $1000 \mathrm{~m}$ ). Ces secteurs sont relativement dispersés sur tout le territoire d'étude avec une présence dans les couronnes nord et sud et à Laval; et dans l'agglomération de Montréal, essentiellement dans quelques secteurs situés dans les arrondissements du centre et de l'est de l'île.

Près du tiers des secteurs (en gris foncé, $n=282,32 \%$ ) se caractérisent par une bonne accessibilité immédiate (distance inférieure à $500 \mathrm{~m}$ d'une aire de jeux), mais avec une moyenne ou forte congestion potentielle (3 premiers quintiles de I'E2SFCA). Bien entendu, ils sont surtout localisés dans le centre de l'agglomération de Montréal. À l'inverse, en gris pâle, sont représentés des secteurs ( $n=178,20,2 \%$ ) principalement localisés dans les banlieues et caractérisés par un éloignement important de l'aire de jeux la plus proche (à $500 \mathrm{~m}$ ou plus), mais des niveaux de congestion potentielle très faibles. Finalement, les secteurs en blanc ( $n=201,22,8 \%$ ) présentent des niveaux d'accessibilité de faible à moyen quant à la proximité, mais aussi des niveaux moyen ou faible quant à la congestion.Ces derniers sont dispersés sur tout sur le territoire. 


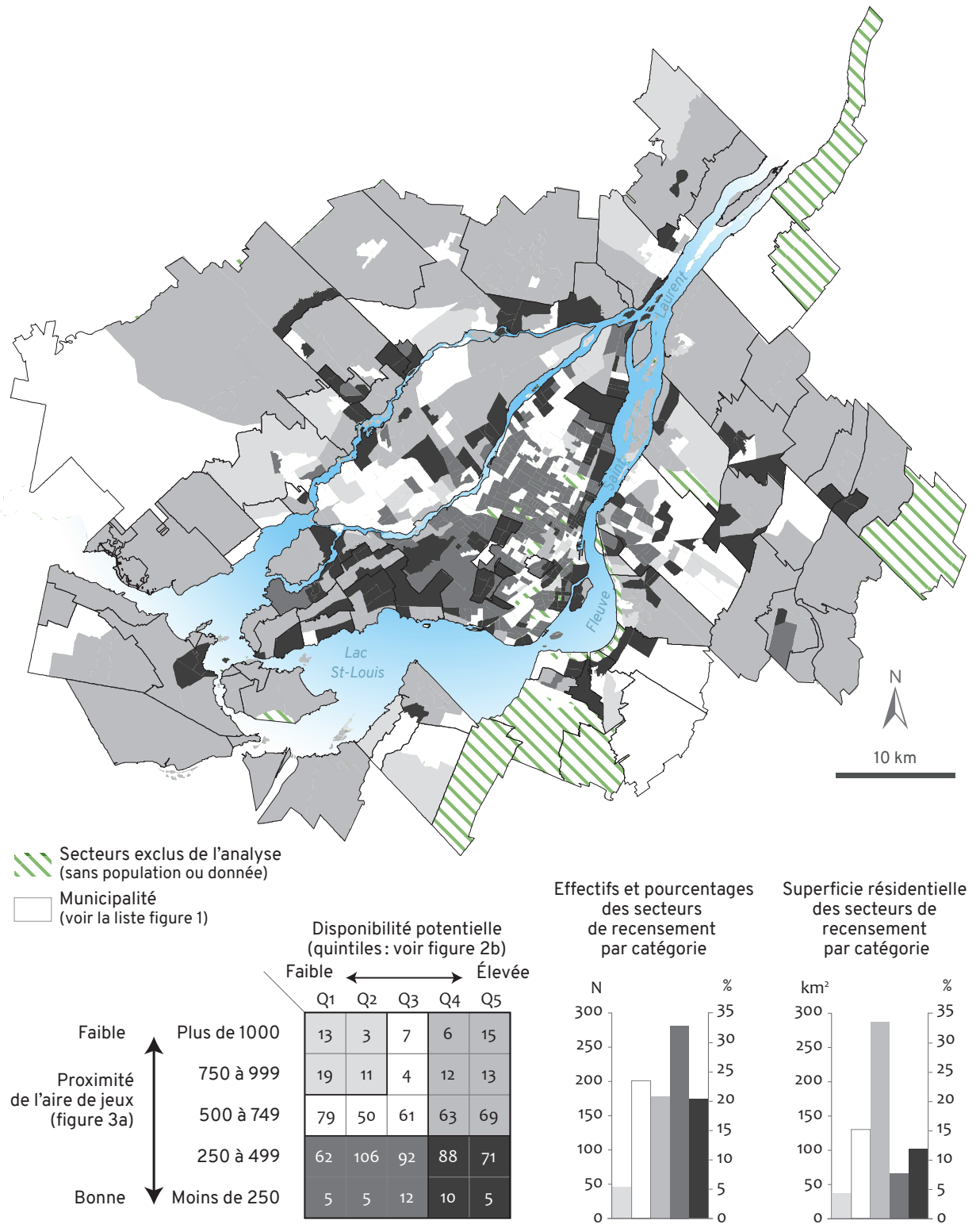

FIGURE 3 Typologie des secteurs de recensement en fonction des deux indicateurs d'accessibilité | Conception: Alvarenga, Apparicio et Ségin 2018 Adaptée par le Département de géographie de l'Université Laval, 2019 


\section{Diagnostic d'équité environne- mentale: régressions multiples et multinomiales}

Les résultats des régressions linéaires multiples avec, comme variables dépendantes, les deux indicateurs d'accessibilité sont reportés au tableau 2. Le premier bloc de variables indépendantes permet de contrôler l'effet des cinq grandes régions de la CMM et confirme les analyses précédentes. Comparativement aux secteurs situés dans l'agglomération de Montréal (catégorie de référence), ceux des autres sous-régions présentent une plus grande distance de l'aire de jeux la plus proche. Par exemple, toutes choses étant égales par ailleurs, un secteur de la couronne nord est en moyenne localisé à $150 \mathrm{~m}$ de plus de l'aire de jeux qu'un secteur de l'agglomération de Montréal. Tous les coefficients sont d'ailleurs significatifs $(P<0,001)$ et s'élèvent à 88 m à Laval, 96 à Longueuil et même 184 dans le reste de la couronne sud. Concernant le second modèle (avec l'E2SFCA comme variable dépendante), seuls les coefficients de la couronne nord $(B=5,25)$ et du reste de la couronne sud $(B=7,98)$ sont significatifs. Dans ces deux zones, la saturation potentielle est donc en moyenne plus faible qu’à Montréal.

Une fois ces variables muettes régionales contrôlées, il est possible de poser un diagnostic d'équité environnementale. Avant d'analyser en détail les coefficients, il convient de mentionner que les valeurs du facteur d'inflation de la variance (VIF) sont toutes inférieures à 5 , signalant ainsi une absence de multicolinéarité excessive entre les variables indépendantes. Élément positif et rassurant, plus la densité d'enfants de moins de 10 ans augmente $(B=-0,04, P<0,001)$, plus la distance jusqu'à l'aire de jeux la plus proche diminue, mais cette diminution reste très modeste. Les familles monoparentales bénéficient aussi d'une meilleure accessibilité à l'aire de jeux la plus proche, plus marquée pour ce groupe: chaque augmentation d'un point de pourcentage de personne à faible revenu par secteur de recensement diminue de 6,24 $\mathrm{m}$ la distance jusqu'à l'aire de jeux la plus proche $(P<0,001)$. Cependant, le coefficient du pourcentage de personnes à faible revenu traduit une situation d'iniquité environnementale, alors qu'aucune association significative n'est observée pour les minorités visibles. Toutefois, le coefficient est relativement faible: chaque augmentation d'un point de pourcentage de personnes à faible revenu augmente de $2,5 \mathrm{~m}$ la distance de l'aire de jeux la plus proche $(P<0,05)$.
Concernant le modèle avec l'E2SFCA, parmi les variables indépendantes relatives aux quatre groupes de population, seuls deux coefficients sont significatifs: les personnes à faible revenu $(B=-0,30, P<0,001)$ et les minorités visibles $(B=-0,08, P<0,05)$. Plus la présence des personnes à faible revenu et des minorités visibles est importante, plus les aires de jeux dans un rayon de $1000 \mathrm{~m}$ sont potentiellement saturées. Toutefois, là encore, les valeurs des coefficients demeurent très faibles, surtout pour les minorités visibles.

Les résultats de la régression logistique multinomiale sont reportés aux tableaux 3 et 4 . II est à noter que la variable des minorités visibles a été exclue du modèle final, car elle s'est avérée non significative. De plus, nous avons choisi la modalité « gris foncé» (proximité élevée, mais disponibilité faible ou moyenne) comme catégorie de référence, car elle regroupe le plus d'observations ( $n=282,32 \%)$. Les valeurs de Khi 2 au tableau 3 permettent de repérer les variables indépendantes les plus importantes du modèle. ${ }^{3}$ Ce sont dans l'ordre: la densité d'enfants et le pourcentage des familles monoparentales suivis des variables muettes pour les régions et, finalement, la variable sur le faible revenu.

L'analyse des rapports de cote $(R C)$ significatifs $(P<0,05)$ (tableau 4) permet d'avancer plusieurs constats intéressants. Rappelons que la catégorie de référence est le gris foncé et qu'elle est surtout concentrée au centre de l'agglomération de Montréal. Par conséquent, il n'est pas étonnant de retrouver des rapports de cote significatifs pour les autres régions, pour la plupart des catégories. En guise d'exemple, comparativement à l'agglomération de Montréal et avec le gris foncé comme catégorie de référence, nous constatons que les secteurs des quatre autres régions ont une plus forte probabilité d'appartenir à la catégorie «gris très pâle » caractérisée par une faible proximité et une faible disponibilité (RC de 12,63, 12,62, 4,45 et 4,19 respectivement pour la couronne nord, le reste de la couronne sud, Laval et l'agglomération de Longueuil). À l'inverse, pour la catégorie «gris très foncé», caractérisée par une très bonne accessibilité selon les deux indicateurs, aucun rapport de cote n'est significatif pour les quatre régions. Autrement dit, cette catégorie n'est pas plus ou moins présente dans ces quatre régions que dans l'agglomération de Montréal.

3 Cette démarche classique en régression logistique multinomiale consiste à comparer un modèle comprenant l'ensemble des variables indépendantes (VI) et un autre comprenant l'ensemble des VI auquel une $\mathrm{VI}$ a été ôtée. Si le test du rapport de vraisemblance entre les deux modèles est significatif, cela signifie que les deux modèles sont différents et que la VI supprimée joue un rôle significatif dans le modèle final. Cette opération est réalisée pour chacune des VI. 
TABLEAU 2 Modèles de régression linéaire multiple pour les deux indicateurs d'accessibilité | Conception: Alvarenga, Apparicio et Séguin, 2018

\begin{tabular}{|c|c|c|c|c|c|c|}
\hline \multirow[t]{2}{*}{ Variable dépendante } & \multicolumn{3}{|c|}{$\begin{array}{l}\text { Proximité de l'aire de jeu la plus proche } \\
\text { (en mètres) }\end{array}$} & \multicolumn{3}{|c|}{$\begin{array}{l}\text { Disponibilité potentielle } \\
\text { (aire de jeu pour } 1000 \text { enfants) }\end{array}$} \\
\hline & Coefficient & $\mathrm{T}$ & VIF & Coefficient & $\mathrm{T}$ & VIF \\
\hline Constante & $655,009 * * *$ & 24,29 & & $30,662^{* * *}$ & 14,03 & \\
\hline Agglomération de Montréal & Réf. & & & Réf. & & \\
\hline Ville de Laval & $87,535 * * *$ & 3,99 & 1,22 & 0,875 & 0,50 & 1,19 \\
\hline Couronne nord & $149,564 * * *$ & 7,03 & 1,48 & $5,247 * * *$ & 3,07 & 1,48 \\
\hline Agglomération de Longueuil & $95,623 * * *$ & 4,56 & 1,22 & 2,138 & 1,27 & 1,17 \\
\hline Reste de la couronne sud & $184,187 * * *$ & 7,94 & 1,44 & $7,976 * * *$ & 4,26 & 1,44 \\
\hline $\begin{array}{l}\text { Densité d'enfants de moins de } 10 \text { ans } \\
\text { (enfants } / \mathrm{km}^{2} \text { ) }\end{array}$ & $-0,040 * * *$ & $-5,39$ & 2,35 & -- & & \\
\hline Familles monoparentales (\%) & $-6,242 * * *$ & $-7,23$ & 2,39 & $-0,027$ & $-0,40$ & 2,35 \\
\hline Personnes à faible revenu (\%) & $2,510 *$ & 2,46 & 4,38 & $-0,303 * * *$ & $-3,99$ & 3,75 \\
\hline Minorités visibles (\%) & 0,635 & 1,19 & 2,45 & $-0,083 *$ & $-1,97$ & 2,35 \\
\hline Nombre de secteurs de recensement ${ }^{a}$ & 867 & & & 868 & & \\
\hline $\mathrm{R}^{2}$ & 0,321 & & & 0,193 & & \\
\hline $\mathrm{R}^{2}$ ajusté & 0,314 & & & 0,186 & & \\
\hline
\end{tabular}

a Relativement aux conditions d'application, les observations avec une valeur de distance de Cook supérieure à 8/N ont été supprimées du modèle final. Cela explique que le nombre d'observations varie d'un modèle à l'autre. ${ }^{* * *} p<0,001 ;{ }^{* *} p<0,01 ;{ }^{*} p<0,05$

L'analyse des variables socioéconomiques révèle que, plus la densité des enfants augmente dans un secteur de recensement, plus la probabilité d'appartenir aux catégories «gris très pâle», «gris pâle » et «gris très foncé» diminue. Autrement dit, plus la densité augmente, plus un secteur risque d'appartenir à la catégorie «gris foncé», soit celle caractérisée par une bonne proximité des aires de jeux, mais fortement saturée. Il en va de même pour les familles monoparentales, excepté pour la catégorie «gris très foncé». Toutefois, plus le pourcentage de personnes à faible revenu augmente dans un secteur, plus la probabilité d'appartenir à un secteur $(\mathrm{RC}=1,05)$ caractérisé par des valeurs moyennes pour les deux indicateurs (secteurs en blanc) augmente.
TABLEAU 3 Variables indépendantes les plus significatives du modèle de régression logistique multinomiale | Conception: Alvarenga, Apparicio et Séguin 2018

\begin{tabular}{|l|c|c|}
\hline & Khi 2 de Wald & P \\
\hline $\begin{array}{l}\text { Densité d'enfants de moins de } \\
10 \text { ans (enfants } / \mathrm{km}^{2} \text { ) }\end{array}$ & 102,64 & $<0,0001$ \\
\hline Familles monoparentales (\%) & 34,36 & $<0,0001$ \\
\hline Ville de Laval & 27,59 & $<0,0001$ \\
\hline Couronne nord & 25,07 & $<0,0001$ \\
\hline Agglomération de Longueuil & 19,07 & 0,0008 \\
\hline Reste de la couronne sud & 14,85 & 0,0050 \\
\hline Personnes à faible revenu (\%) & 14,25 & 0,0065 \\
\hline AlC & 2149,95 & \\
\hline R2 (Cox and Snell) & 0,492 & \\
\hline
\end{tabular}


TABLEAU 4 Coefficients et rapports de cote de la régression logistique multinomiale (catégorie de référence: gris foncé) Conception : Alvarenga, Apparicio et Séguin, 2018

\begin{tabular}{|c|c|c|c|c|c|c|c|c|c|c|}
\hline \multirow[t]{2}{*}{ Catégoriea } & Coefficient & $\mathrm{RC}$ & \multicolumn{2}{|c|}{$\mathrm{RC}(95 \%)$} & $\mathrm{P}$ & Coefficient & $\mathrm{RC}$ & \multicolumn{2}{|c|}{$\mathrm{RC}(95 \%)$} & $P$ \\
\hline & \multicolumn{5}{|c|}{ Ville de Laval } & \multicolumn{5}{|c|}{ Densité d'enfants de moins de 10 ans au km² } \\
\hline Gris très pâle & 1,494 & 4,45 & 1,33 & 14,92 & 0,015 & $-0,001$ & 1,00 & 1,00 & 1,00 & 0,047 \\
\hline Blanc & 1,537 & 4,65 & 2,07 & 10,45 & 0,000 & 0,000 & 1,00 & 1,00 & 1,00 & 0,001 \\
\hline Gris pâle & 0,848 & 2,34 & 0,93 & 5,88 & 0,072 & $-0,004$ & 1,00 & 1,00 & 1,00 & $<, 0001$ \\
\hline \multirow[t]{2}{*}{ Gris très foncé } & $-0,373$ & 0,69 & 0,26 & 1,81 & 0,450 & $-0,001$ & 1,00 & 1,00 & 1,00 & $<, 0001$ \\
\hline & \multicolumn{5}{|c|}{ Couronne nord } & \multicolumn{5}{|c|}{ Familles monoparentales (\%) } \\
\hline Gris très pâle & 2,536 & 12,63 & 2,95 & 53,99 & 0,001 & $-0,043$ & 0,96 & 0,91 & 1,01 & 0,086 \\
\hline Blanc & 2,316 & 10,13 & 3,24 & 31,72 & $<, 0001$ & $-0,057$ & 0,95 & 0,92 & 0,97 & $<, 0001$ \\
\hline Gris pâle & 1,713 & 5,54 & 1,70 & 18,07 & 0,005 & $-0,060$ & 0,94 & 0,91 & 0,98 & 0,003 \\
\hline \multirow[t]{2}{*}{ Gris très foncé } & 0,953 & 2,59 & 0,81 & 8,31 & 0,109 & 0,023 & 1,02 & 0,99 & 1,05 & 0,135 \\
\hline & \multicolumn{5}{|c|}{ Agglomération de Longueuil } & \multicolumn{5}{|c|}{ Personnes à faible revenu (\%) } \\
\hline Gris très pâle & 1,432 & 4,19 & 1,36 & 12,93 & 0,013 & 0,013 & 1,01 & 0,95 & 1,08 & 0,671 \\
\hline Blanc & 0,976 & 2,65 & 1,26 & 5,61 & 0,011 & 0,049 & 1,05 & 1,02 & 1,08 & 0,001 \\
\hline Gris pâle & 0,255 & 1,29 & 0,55 & 3,04 & 0,561 & 0,028 & 1,03 & 0,98 & 1,08 & 0,246 \\
\hline \multirow[t]{2}{*}{ Gris très foncé } & $-0,435$ & 0,65 & 0,29 & 1,45 & 0,293 & $-0,006$ & 0,99 & 0,96 & 1,03 & 0,747 \\
\hline & \multicolumn{5}{|c|}{ Reste de la couronne sud } & & & & & \\
\hline Gris très pâle & 2,535 & 12,62 & 1,97 & 80,92 & 0,008 & & & & & \\
\hline Blanc & 2,548 & 12,78 & 2,75 & 59,36 & 0,001 & & & & & \\
\hline Gris pâle & 2,196 & 8,99 & 1,93 & 42,00 & 0,005 & & & & & \\
\hline Gris très foncé & 1,503 & 4,50 & 0,96 & 20,98 & 0,056 & & & & & \\
\hline
\end{tabular}

aVoir la figure 3: RC = rapport de cote. $\mathrm{RC}(95 \%)=$ intervalle de rapport de cote à $95 \%$.

\section{Discussion des résultats}

L'utilisation de deux indicateurs d'accessibilité spatiale relatifs à la proximité immédiate et à la disponibilité (saturation ou congestion) nous a permis de démontrer que: dans les zones de banlieue, les enfants résident plus loin de l'aire de jeux la plus proche (souvent à plus $750 \mathrm{~m}$ ) qui est par ailleurs faiblement saturée (peu d'utilisateurs potentiels dans un rayon de $1000 \mathrm{~m}$ ) alors que, dans le centre de l'agglomération de Montréal, les enfants résident près d'une aire de jeux (souvent à moins de 500 m), mais qui est potentiellement saturée. Ces résultats viennent corroborer des études antérieures menées à Baltimore et à Los Angeles sur les parcs (Boone et al., 2009; Sister et al., 2010). Notons toutefois que la proximité ne semble pas être un problème majeur puisque $90 \%$ des enfants de la CMM ont accès à une aire de jeux à moins de $800 \mathrm{~m}$.
Ces résultats méritent cependant d'être discutés à la lumière des caractéristiques du tissu résidentiel d'insertion des parcs et des notions de capacité d'utilisation et de densité fonctionnelle proposées par Cohen et al. (2010). Tout d'abord, les effets de la proximité de l'aire de jeu la plus proche risquent de varier en fonction du milieu résidentiel. Dans les zones résidentielles de banlieue, les enfants jouent beaucoup dans les arrière-cours des maisons individuelles - où sont parfois installés des modules de jeu - et même dans les rues résidentielles à très faible trafic, ce qui vient compenser la plus faible proximité des aires de jeu. Autrement dit, l'aire de jeu n'est pas toujours le principal lieu extérieur de pratique d'activités récréatives, physiques et de socialisation pour l'enfant. Par contre, dans les quartiers centraux à forte densité résidentielle où la plupart des enfants ne disposent pas d'une arrière-cour, le rôle de l'aire de jeux et de sa proximité peut s'avérer beaucoup plus important. 
Deuxièmement, la saturation des parcs peut aussi avoir des effets différents selon le milieu. Bien qu'il n'existe pas de seuil établi quant au nombre d'utilisateurs potentiels d'une aire de jeux, des auteurs comme Cohen et al. (2010) mettent de l'avant deux concepts intéressants pour les aires de jeux: la capacité du lieu (carrying capacity) et la densité fonctionnelle (functional density). La première renvoie au nombre maximum d'utilisateurs que peut tolérer une aire de jeux avant d'entraîner une dégradation accélérée des équipements; la seconde renvoie au nombre d'utilisateurs idéal de l'aire de jeux afin d'optimiser la satisfaction des enfants (ni peu ni trop fréquenté). Or, il est probable que dans les quartiers centraux, la saturation élevée conduise à une dégradation accélérée des modules des aires de jeux et décourage certains utilisateurs potentiels de s'y rendre, surtout aux moments de grande affluence (comme les fins de semaine ou les soirs de beau temps). À l'inverse, les aires de jeux en banlieue risquent d'être sous-utilisées: un enfant n'ira pas y jouer si peu de ses pairs y sont présents.

Pour terminer, mentionnons que sur le plan de l'aménagement, l'approche développée ici-qui croise deux indicateurs d'accessibilité et cartographie les secteurs de recensement en fonction des niveaux de saturation et de proximité des aires de jeux - peut s'avérer un outil très intéressant pour les planificateurs urbains. En effet, elle a permis de déterminer 46 secteurs éloignés des aires de jeux, lesquelles sont d'ailleurs potentiellement saturées.

\section{Conclusion}

Bien que les résultats diffèrent selon les groupes étudiés, notre diagnostic d'équité environnementale a montré qu'il n'existe pas d'iniquités flagrantes en termes d'accessibilité aux aires de jeux, sur le territoire de la CMM. Les résultats des modèles de régression ont montré que certains groupes bénéficient même d'une situation favorable, comme les enfants et les familles monoparentales, qui vivent en moyenne plus proche des aires de jeux. En revanche, les personnes à faible revenu vivent dans des milieux plus éloignés des aires de jeux, qui sont d'ailleurs plus saturées. Par contre, les coefficients de régression sont très faibles. Enfin, pour les populations appartenant aux minorités visibles, les résultats ne permettent pas de conclure à une situation d'iniquité, car ils ne sont pas significatifs.

La typologie basée sur le croisement des deux indicateurs d'accessibilité (distance de l'aire de jeux la plus proche et E2SFCA) a révélé l'existence de 46 secteurs (sur un total de 881) caractérisés par une faible accessibilité selon les deux indicateurs. Ces secteurs mériteraient une attention particulière de la part des planificateurs urbains afin de vérifier s'ils nécessitent ou non l'implantation de nouvelles aires de jeux.

Il convient de dégager plusieurs limites à cette étude et plusieurs pistes de recherche. Nous n'avons pas tenu compte de la qualité du milieu d'insertion des aires de jeux, qui peut influencer grandement à la fois leur accessibilité et leur utilisation. Des indicateurs relatifs à la marchabilité des rues, la criminalité et le sentiment de sécurité, la densité du trafic, ou encore le bruit autour des aires mériteraient d'être intégrés dans l'évaluation de leur accessibilité. En outre, le calcul des mesures d'accessibilité pourrait être réalisé selon d'autres modes de transport actif, notamment le vélo (Apparicio et al., 2017). Nous n'avons pas non plus intégré des indicateurs relatifs à la qualité et au design des équipements dans les aires de jeux. Or, relativement à ces critères, certains auteurs signalent que les aires de jeux peuvent être moins attrayantes, voire inintéressantes, pour les enfants (Maxey, 1999; Veitch et al., 2007).

Finalement, le recours à la mesure du E2SFCA s'est avéré fort utile pour évaluer la saturation potentielle des aires de jeux. Toutefois, il est très difficile d'établir des seuils à partir desquels la saturation pourrait être jugée problématique. Des études qualitatives utilisant notamment des observations de terrain, des groupes de discussion, mais aussi des entretiens avec des ergonomes, des spécialistes de design d'aires de jeux et des gestionnaires des parcs permettraient d'explorer les notions de capacité d'utilisateurs (carrying capacity) et de densité fonctionnelle (functional density) des aires, puis de formuler des hypothèses quant à ces seuils, et ce, dans une logique de planification.

\section{Bibliographie}

ABERCROMBIE, Lauren C., SALLIS, James F., CONWAY, Terry L., FRANK, Lawrence D., SAELENS, Brian E. et al. (2008) Income and racial disparities in access to public parks and private recreation facilities. American Journal of Preventive Medicine, vol. 34, no1, p. 9-15.

APPARICIO, Philippe, CLOUTIER, Marie-Soleil, SÉGUIN, AnneMarie et ADES, Josefina (2010) Accessibilité spatiale aux parcs urbains pour les enfants et injustice environnementale. Exploration du cas montréalais. Revue internationale de géomatique, vol. 20, n³, p. 363-389. 
APPARICIO, Philippe, GELB, Jérémy, DUBÉ, Anne-Sophie, KINGHAM, Simon, GAUVIN, Lise et al. (2017) The approaches to measuring the potential spatial access to urban health services revisited: Distance types and aggregation-error issues. International Journal of Health Geographics, vol. 16, n०32, p. 1-24.

APPARICIO, Philippe, SÉGUIN, Anne-Marie et DUBÉ, Jean (2016) Spatial distribution of vegetation in and around city blocks on the Island of Montreal: A double environmental inequity? Applied Geography, vol. 76, p. 128-136.

BELL, Scott, WILSON, Kathi, BISSONNETTE, Laura et SHAH, Tayyab (2013) Access to primary health care: Does neighborhood of residence matter? Annals of the Association of American Geographers, vol. 103, no1, p. 85-105.

BOONE, Christopher, BUCKLEY, Geoffrey, GROVE, J. Morgan et SISTER, Chona (2009) Parks and people: An environmental justice inquiry in Baltimore, Maryland. Annals of the Association of American Geographers, vol. 99, n4, p. 767-787.

BURDETTE, Hillary L. et WHITAKER, Robert C. (2004) Neighborhood playgrounds, fast food restaurants, and crime: Relationships to overweight in low-income preschool children. Preventive Medicine, vol. 38, no1, p. 57-63.

CARRIER, Mathieu, APPARICIO, Philippe, KESTENS, Yan, SÉGUIN, Anne-Marie, PHAM, Hien et al. (2016) Application of a global environmental equity Index in Montreal: Diagnostic and further implications. Annals of the American Association of Geographers, vol. 106, n$^{\circ} 6$, p. 1268-1285.

CHIESURA, Anna (2004) The role of urban parks for the sustainable city. Landscape and Urban Planning, vol. 68, n01, p. 129-138.

COEN, Stephanie E. et ROSS, Nancy A. (2006) Exploring the material basis for health: Characteristics of parks in Montreal neighborhoods with contrasting health outcomes. Health \& Place, vol. 12, nº4, p. 361-371.

COHEN, Deborah A., MARSH, Terry, WILLIAMSON, Stephanie, DEROSE, Kathryn Pitkin, MARTINEZ, Homero et al. (2010) Parks and physical activity: Why are some parks used more than others? Preventive Medicine, vol. 50, p. S9-S12.

CRADOCK, Angie L., KAWACHI, Ichiro, COLDITZ, Graham A., HANNON, Cynthia, MELLY, Steven J. et al. (2005) Playground safety and access in Boston neighborhoods. American Journal of Preventive Medicine, vol. 28, n4, p. 357-363.

CUTTS, Bethany B., DARBY, Kate J., BOONE, Christopher G. et BREWIS, Alexandra (2009) City structure, obesity, and environmental justice: An integrated analysis of physical and social barriers to walkable streets and park access. Social Science \& Medicine, vol. 69, n॰9, p. 1314-1322.

CZALCZYNSKA-PODOLSKA, Magdalena (2014) The impact of playground spatial features on children's play and activity forms: An evaluation of contemporary playgrounds' play and social value. Journal of Environmental Psychology, vol. 38, p. 132-142.
ELLAWAY, Anne, KIRK, Alison, MACINTYRE, Sally et MUTRIE, Nanette (2007) Nowhere to play? The relationship between the location of outdoor play areas and deprivation in Glasgow. Health \& Place, vol. 13, n², p. 557-561.

FONTAINE, Nicolas (2011) La contribution aux fins de parc, terrains de jeux et espaces naturels: où en sommes-nous? Bulletin de l'Observatoire québécois du loisir, vol. 9, n², p. 1-6.

GILES-CORTI, Billie, BROOMHALL, Melissa H., KNUIMAN, Matthew, COLLINS, Catherine, DOUGLAS, Kate et al. (2005) Increasing walking: How important is distance to, attractiveness, and size of public open space? American Journal of Preventive Medicine, vol. 28, n², p. 169-176.

HECKERT, Megan et ROSAN, Christina D. (2016) Developing a green infrastructure equity index to promote equity planning. Urban Forestry \& Urban Greening, vol. 19, p. 263-270.

HODGSON, M. John et HEWKO, Jared (2003) Aggregation and surrogation error in the p-median model. Annals of Operations Research, vol. 123, n01, p. 53-66.

JONES, Andy, HILLSDON, Melvyn et COOMBES, Emma (2009) Greenspace access, use, and physical activity: Understanding the effects of area deprivation. Preventive Medicine, vol. 49, n6, p. 500-505.

KACZYNSKI, Andrew T., POTWARKA, Luke R., SMALE, Bryan J. A. et HAVITZ, Mark E. (2009) Association of parkland proximity with neighborhood and park-based physical activity: Variations by gender and age. Leisure Sciences, vol. 31, n², p. 174-191.

KARSTEN, Lia (2002) Mapping childhood in Amsterdam: The spatial and social construction of children's domains in the city. Tijdschrift voor economische en sociale geografie, vol. 93, n०3, p. 231-241.

LOUKAITOU-SIDERIS, Anastasia et STIEGLITZ, Orit (2002) Children in Los Angeles parks: A study of equity, quality and children's satisfaction with neighbourhood parks. Town Planning Review, vol. 73, n4, p. 467-488.

LOVASI, Gina S., JACOBSON, Judith S., QUINN, James W., NECKERMAN, Kathryn M., ASHBY-THOMPSON, Maxine N. et al. (2011) Is the environment near home and school associated with physical activity and adiposity of urban preschool children? Journal of Urban Health, vol. 88, n6, p. 1143-1157.

LOW, Setha, TAPLIN, Dana et SCHELD, Suzanne (2009) Rethinking urban parks: Public space and cultural diversity. Austin, University of Texas Press.

LUO, Wei et QI, Yi (2009) An enhanced two-step floating catchment area (E2SFCA) method for measuring spatial accessibility to primary care physicians. Health \& place, vol. 15 , n०4, p. 1100-1107. 
LUO, Wei et WANG, Fahui (2003) Measures of spatial accessibility to health care in a GIS environment: Synthesis and a case study in the Chicago region. Environment and Planning B: Planning and Design, vol. 30, n6, p. 865-884.

MAROKO, Andrew R., MAANTAY, Juliana A., SOHLER, Nancy L., GRADY, Kristen L. and ARNO, Peter S. (2009) The complexities of measuring access to parks and physical activity sites in New York City: A quantitative and qualitative approach. International Journal of Health Geographics, vol. 8, n³4, p. 1-23.

MAXEY, Ian (1999) Playgrounds: From oppressive spaces to sustainable places? Built Environment, vol. 25, n01, p. 18-24.

MCGRAIL, Matthew R. et HUMPHREYS, John S. (2009) Measuring spatial accessibility to primary care in rural areas: Improving the effectiveness of the two-step floating catchment area method. Applied Geography, vol. 29, n4, p. 533-541.

MILLER, Lucy Jane, SCHOEN, Sarah A., CAMARATA, Stephen M. MCCONKEY, John, KANICS, Ingrid M. et al. (2017) Play in natural environments: A pilot study quantifying the behavior of children on playground equipment. Journal of Occupational Therapy, Schools, \& Early Intervention, vol. 10 n³, p. 1-19.

NGUI, André Ngamini et APPARICIO, Philippe (2011) Optimizing the two-step floating catchment area method for measuring spatial accessibility to medical clinics in Montreal. BMC Health Services Research, vol. 11, no166, p. 1-12.

PETERS, Karin, ELANDS, Birgit et BUIJS, Arjen (2010) Social interactions in urban parks: Stimulating social cohesion? Urban Forestry \& Urban Greening, vol. 9, n², p. 93-100.

PHAM, Thi-Thanh-Hien, APPARICIO, Philippe, SÉGUIN, AnneMarie, LANDRY, Shawn et GAGNON, Martin (2012) Spatial distribution of vegetation in Montreal: An uneven distribution or environmental inequity? Landscape and Urban Planning, vol. 107, n³, p. 214-224.

POOLE, Robin et MOON, Graham (2017) What is the association between healthy weight in 4-5-year-old children and spatial access to purposefully constructed play areas? Health \& Place, vol. 46, p. 101-106.

POTWARKA, Luke R., KACZYNSKI, Andrew T. et FLACK, Andrea L. (2008) Places to play: Association of park space and facilities with healthy weight status among children. Journal of Community Health, vol. 33, n5, p. 344-350.

ROEMMICH, James N., EPSTEIN, Leonard H., RAJA, Samina, YIN, Li, ROBINSON, Jodie et al. (2006) Association of access to parks and recreational facilities with the physical activity of young children. Preventive Medicine, vol. 43, nº, p. 437-441.

SALLIS, James F., MCKENZIE, Thomas L., ELDER, John P., BROYLES, Shelia L. et NADER, Philip R. (1997) Factors parents use in selecting play spaces for young children. Archives of Pediatrics \& Adolescent Medicine, vol. 151, n4, p. 414-417.
SÉGUIN, Anne-Marie et APPARICIO, Philippe (2013) Justice environnementale. Cahiers de géographie du Québec, vol. 57, n0161, p. 211-214 [En ligne]. https://www.erudit.org/fr/revues/ cgq/2013-v57-n161-cgq01387/1024901ar.pdf

SINGH, Gopal K., SIAHPUSH, Mohammad et KOGAN, Michael D. (2010) Neighborhood socioeconomic conditions, built environments, and childhood obesity. Health Affairs, vol. 29, n०3, p. 503-512.

SISTER, Chona, WOLCH, Jennifer et WILSON, John (2010) Got green? Addressing environmental justice in park provision. GeoJournal, vol. 75, no3, p. 229-248.

SMOYER-TOMIC, Karen E., HEWKO, Jared N. et HODGSON, M John (2004) Spatial accessibility and equity of playgrounds in Edmonton, Canada. The Canadian Geographer, vol. 48, n³, p. 287-302.

TALEN, Emily et ANSELIN, Luc (1998) Assessing spatial equity: An evaluation of measures of accessibility to public playgrounds. Environment and Planning A, vol. 30, n4, p. 595-613.

TAPPE, Karyn A., GLANZ, Karen, SALLIS, James F., ZHOU, Chuan et SAELENS, Brian E. (2013) Children's physical activity and parents' perception of the neighborhood environment: Neighborhood impact on kids study. International Journal of Behavioral Nutrition and Physical Activity, vol. 10, n¹, p. 39.

TIMPERIO, Anna, GILES-CORTI, Billie, CRAWFORD, David, ANDRIANOPOULOS, Nick, BALL, Kylie et al. (2008) Features of public open spaces and physical activity among children: Findings from the CLAN study. Preventive Medicine, vol. 47, n०5, p. 514-518.

TUCKER, Patricia, IRWIN, Jennifer D., GILLILAND, Jason, HE, Meizi, LARSEN, Kristian et HESS, Paul (2009) Environmental influences on physical activity levels in youth. Health \& Place, vol. $15, n^{01}$, p. 357-363

VAN DEN BERG, Agnes E., MAAS, Jolanda, VERHEIJ, Robert A. et GROENEWEGEN, Peter P. (2010) Green space as a buffer between stressful life events and health. Social Science \& Medicine, vol. 70, n॰8, p. 1203-1210.

VEITCH, Jenny, SALMON, Jo et BALL, Kylie (2007) Children's perceptions of the use of public open spaces for active freeplay. Children’s Geographies, vol. 5, n4, p. 409-422.

VEUGELERS, Paul, SITHOLE, Fortune, ZHANG, Sharon et MUHAJARINE, Nazeem (2008) Neighborhood characteristics in relation to diet, physical activity and overweight of Canadian children. Pediatric Obesity, vol. 3, n³, p. 152-159.

VILLE DE MONTRÉAL (2008) Pour grandir à Montréal: politique familiale de Montréal 2008. Montréal, Direction des communications et des relations avec les citoyens.

WALKER, Gordon (2012) Environmental justice: Concepts, evidence and politics. Londres, Routledge. 
WAN, Neng, ZHAN, F. Benjamin, ZOU, Bin et CHOW, Edwin (2012) A relative spatial access assessment approach for analyzing potential spatial access to colorectal cancer services in Texas. Applied Geography, vol. 32, n², p. 291-299.

WIEBE, Isabel (2010) L'accessibilité spatiale et les pratiques d'utilisation des services et équipements pour les enfants dans l'arrondissement Rosemont-La Petite-Patrie. Québec, Université du Québec, Institut national de la recherche scientifique, Programme d’études urbaines, mémoire de maîtrise non publié.

WOLCH, Jennifer, JERRETT, Michael, REYNOLDS, Kim, MCCONNELL, Rob, CHANG, Roger et al. (2011) Childhood obesity and proximity to urban parks and recreational resources: A longitudinal cohort study. Health \& Place, vol. 17, n01, p. 207-214.

WOLCH, Jennifer, WILSON, John P. et FEHRENBACH, Jed (2005) Parks and park funding in Los Angeles: An equity-mapping analysis. Urban Geography, vol. 26, n¹, p. 4-35. 